\title{
An Economic Phase-Mdulation to Intensity-Modulation Converter
}

\author{
Ching-Hung Chang ${ }^{1 *}$, Jui-Hsuan Chang ${ }^{2}$, Yin-Liang Liao², Meng Chun Tseng ${ }^{2}$ \\ ${ }^{1}$ Department of Electrical Engineering, National Chiayi University; ${ }^{2}$ Department of Computer Science and Information Engineering \\ National Chiayi University. \\ Email: ${ }^{*}$ chchang@mail.ncyu.edu.tw
}

Received September 2013

\begin{abstract}
A novel phase-modulation to intensity-modulation (PM-to-IM) converter is proposed and experimentally demonstrated basing on a vertical-cavity surface-emitting laser (VCSEL). Comparing with the published schemes, in which the employed DI, FBG, OBPF or dispersion devices can only process a certain phase-modulated RF signal, the proposed scheme can achieve the conversion process in an economic and resourceful manner. To be the first one of achieving such PM-to-IM conversion process by a VCSEL, the feasibility of the proposal is experimentally demonstrated with an open eye diagram when it is employed to convert phase-modulated RF signal.
\end{abstract}

Keywords: Long-Reach PON; Phase Modulation; Vertical-Cavity Surface-Emitting Laser

\section{Introduction}

With standard single mode fiber (SMF) and conventional optical external intensity modulators, such as MachZehnder modulator (MZM), microwave signal can be easily modulated with an optical carrier and communicated to its destination [1,2]. Nevertheless, an optimized DC bias is required to add on the MZM to obtain high quality optical microwave signal. Different with utilizing the MZM to generate the optical microwave signals, an optical phase modulator (PM) can simply utilize the optical phase shifting to record the microwave states without a need of a DC bias to control the operating point of the electrical to optical conversion processes, so it will not suffer from the DC bias-drifting problem [3]. Besides, such PM systems can efficiently against unwanted selfphase-modulation (SPM) and cross-phase-modulation (XPM) effects as well as eliminate cross-gain-modulation (XGM) effects since the envelope of the generated phasemodulated signal is constant $[4,5]$.

In an optical phase modulation system, the PM signal with a constant envelope needs to be converted into IM signal format before being detected by a PD which only can detect the variation of the optical intensity. Traditionally, such phase modulation to intensity modulation (PMto-IM) conversion process can be achieved by inserting a delay line interferometer (DI) in front of the PD. Nevertheless, the DI itself is sophisticated and expensive.

\footnotetext{
*Corresponding author.
}

Adding it into an optical transport link will significantly reduce the advancements of the PM scheme. In order to overcome this drawback, multiple PM-to-IM conversion schemes have been developed basing a fiber Bragg grating (FBG) [6-8] or an optical band-pass filter (OBPF) $[9,10]$. By reflecting or filtering out one of the +1 or -1 sideband of the received optical phase-modulated signal, a PD will be able to directly convert the received signal back into electrical domain. In addition to filter out one of the sideband, an optical PM-to-IM conversion scheme, is developed based on a dedicated dispersive devices in which are employed to introduce a dispersion-induced PM-to-IM conversion [11-13]. Under these structures, the overall construction cost could be reduced. However, utilizing the fiber dispersion to achieve such PM-to-IM conversion requires a high wavelength stability and a dedicated dispersion value to maximum PM-to-IM conversion. It is not flexible in a WDM transport system. In order to provide an economic and simplify structure to achieve the PM-to-IM conversion process with adjustable microwave frequency range, a novel PM-to-IM converter is proposed.

In this proposal, a vertical-cavity surface-emitting laser (VCSEL) and an optical circulator (OC) are composed as a tunable optical notch amplifier and is utilized to achieve the PM-to-IM conversion process by boosting up the +1 or -1 sideband of the phase-modulated signal. Experimental results proof that the frequency tuning 
range of the notch amplifier is more than $300 \mathrm{GHz}$. This proposal is shown to be an outstanding candidate to achieve long-distance RoF transport systems.

\section{Operation Principle and Experimental Results}

The experimental setup of the proposed tunable PM-toIM converter is shown in the center yellow box of Figure 1, where an OC, a polarization controller (PC) and a commercial VCSEL are employed. In this setup, an optical carrier is firstly phase-modulated with a microwave signal via a PM and then injected into the tunable PMto-IM converter to verify the converter performance. The injected lightwave will be routed and fed into the VCSEL by the OC. Subsequently, the reflected lightwave will be routed by the OC again and then fed into a PD. In this structure, if the +1 sideband of the injected phase-modulated signal, as shown in the insert (i) of the Figure 1, is aligned with the lasing wavelength of the VCSEL an optical amplification process will be introduced into the +1 sideband, as shown in the insert (ii) of the Figure 1. This is because that when the photons of the +1 sideband propagate through and back the laser cavity of the VCSEL, they will hit excited electrons inside the cavity and thus forcing new photon to be emitted at the same wavelength. Once the VCSEL is lasing, the stimulated emission will dominate over stimulated absorption producing an optical gain for the +1 sideband. As shown in the insert (iii) of the Figure 1, the beating amplitude of the center carrier and the +1 sideband will therefore larger than that of the center carrier and the -1 sideband at the PD so the PD can successfully convert the phase-modulated signal back into electronic domain. In order to verify the proposal, a $1.25 \mathrm{Gbps} / 10 \mathrm{GHz}$ signal is experimentally phase modulated with the downstream before being detected by a PD. In an optical phase modulation system, if there is only a single-frequency sinusoidal signal with zero initial phase is modulated, the electrical field of a phase-modulated optical carrier $e_{P M}(t)$ can be expressed as [14]:

$$
e_{P M}(t)=e_{0} \sum_{n=-\infty}^{+\infty} J_{n}\left(\beta_{P M} \nVdash_{e}\right) \cos \left[\left(w_{0}+n w_{m}\right) t+\frac{1}{2} n\right]
$$

where the $e_{0}$ and $w_{0}$ are the amplitude and the angular frequency of the optical carrier. $J_{n}(-)$ denotes the nthorder Bessel function of the first kind. $\beta_{P M}$ is the phase modulation index (radians per volt). $V_{e}$ and $w_{m}$ are the amplitude and angular frequency of the modulating signal. For simplicity, the argument $\left(\beta_{P M} V_{e}\right)$ will be omitted in the remainder of this paper and only the first-order upper and lower sidebands are considered. The small signal assumption of (1) can be further simplified as [25]:

$$
\begin{aligned}
e_{P M}(t)= & e_{0}\left\{J_{0} \cos w_{0} t+J_{1} \cos \left[\left(w_{0}+w m\right) t+\frac{\pi}{2}\right]\right. \\
& \left.+J_{-1} \cos \left[\left(w_{0}-w_{m}\right) t-\frac{\pi}{2}\right]\right\}
\end{aligned}
$$

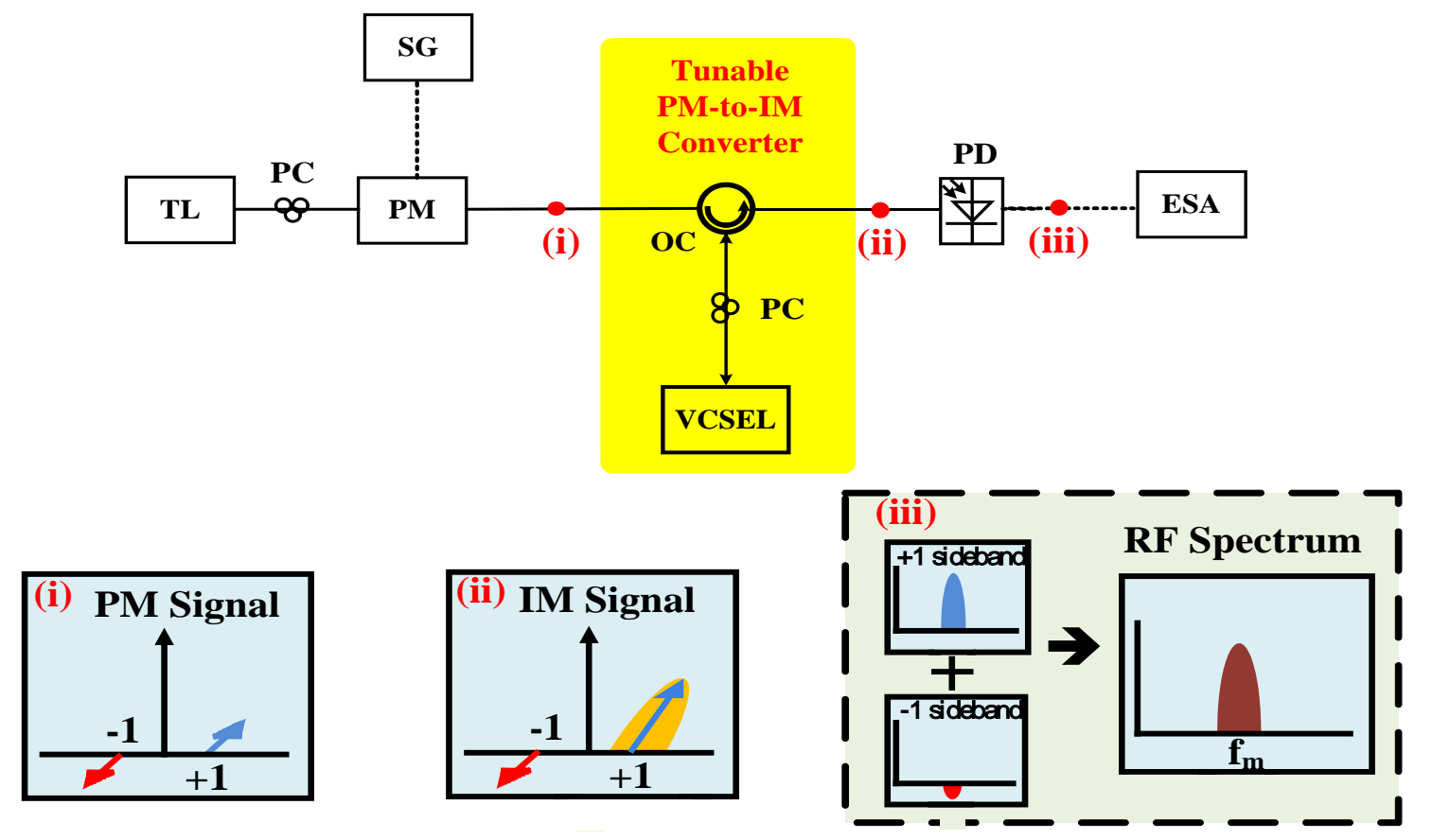

Figure 1. Schematic diagram of the proposed tunable PM-to-IM converter basing on a vertical-cavity surface-emitting laser. (TL: tunable laser, PC: polarization controller, PM: phase modulator, OC: optical circulator, VCSEL: vertical-cavity surface-emitting laser, PD: photodetector, SG: signal generator, ESA: electronic spectrum analyzer). 
For Bessel function, the Jn is equal to $-j-n$ when $n$ is odd. From (2), we can find that if the phase modulated signal is directly injected into a PD, the center carrier will individually beats with +1 and -1 sidebands, resulting in two electrical signals with the same amplitude, the same frequency but different phase. So the PD will not have any output signal. Nevertheless, when the phasemodulated signal is injected into the proposed tunable IM-to-PM converter, the optical intensity of the +1 sideband will be boosted up. As shown in Figures 2 and 3, the optical power variation between the +1 and -1 sidebands is significantly promoted from $0 \mathrm{~dB}$ to $26 \mathrm{~dB}$. A clear eye diagram and obvious electronic spectrum are shown proof in Figure 3. This means that the proposed tunable PM-to-IM converter can successfully modify the received phase-modulated signal back into intensitymodulation format.

\section{Conclusion}

In this paper, a novel PM-to-IM converter is proposed

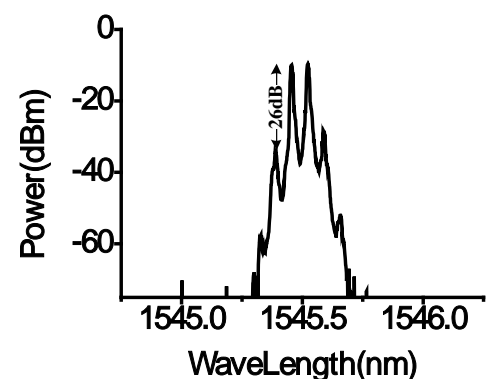

Figure 2. The optical spectrum of phase-modulated RF signal.

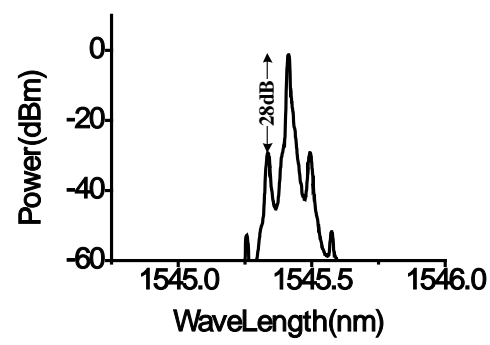

Figure 3. The optical spectrum of phase-modulated RF signal after passing though the PM-to-IM converter.

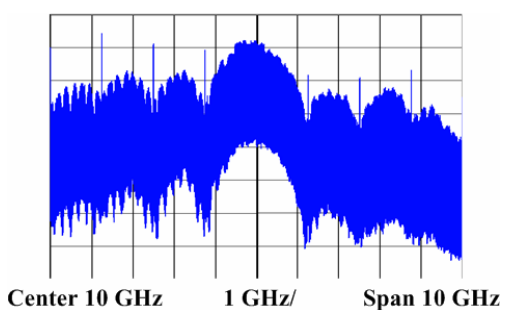

Figure 4. The measured $1.25 \mathrm{Gbps} / 10 \mathrm{GHz}$ downstream signal spectrum. and experimentally demonstrate for optical phase-modulated RoF transport systems. Comparing with the published PM-to-IM conversion schemes, which employ DI, FBG, OBPF or dispersion devices to achieve this target, the proposed scheme can economically and efficiently convert a phase-modulated signal back into intensitymodulated signal by a VCSEL. This is the first one to achieve PM-to-IM conversion process by a VCSEL. The feasibility of the proposal is experimentally demonstrated with open eye diagrams when it is employed to convert phase-modulated RF signal.

\section{REFERENCES}

[1] A. Chiuchiarelli, M. Presi and E. Ciaramella, "Effective Architecture For $10 \mathrm{~Gb} / \mathrm{s}$ Upstream WDM-PONs Exploiting Self-Seeding and External Modulation,” OFC/NFOEC Technology, 2012, pp. 1-3.

[2] T. Shao, F. Paresys, Y. L. Guennec, G. Maury, N. Corrao and B. Cabon, "Convergence of $60 \mathrm{GHz}$ Radio over Fiber and WDM-PON Using Parallel Phase Modulation with a Single Mach-Zehnder Modulator,” Journal of Lightwave Technology, Vol. 30, No. 17, 2012, pp. 2824-2831. http://dx.doi.org/10.1109/JLT.2012.2205370

[3] M. J. La Gasse and S. Thaniyavarn, "Bias-Free High-Dynamic-Rangephase-Modulated Fiber-Optic Link,” IEEE Photonics Technology Letters, Vol. 9, No. 5, 1997, pp. 681-683. http://dx.doi.org/10.1109/68.588207

[4] C. W. Chow and C. H. Yeh, "Signal Remodulation without Power Sacrifice for Carrier Distributed Hybrid WDMTDM PONs Using PolSK,” Optics Communications, Vol. 282, No. 7, 2009, pp. 1294-1297. http://dx.doi.org/10.1016/j.optcom.2008.12.015

[5] J. P. Yao, G. Maury, Y. L. Guennec and B. Cabon, “AllOptical Subcarrier Frequency Conversion Using an Electro-Optic Phase Modulator,” IEEE Photonics Technology Letters, Vol. 17, 2005, pp. 2427-2429.

[6] W. Li, “A Wideband Frequency Tunable Optoelectronic Oscillator Incorporating a Tunable Microwave Photonic Filter Based on Phase-Modulation to Intensity-Modulation Conversion Using a Phase-Shift Fiber Bragg Grating,” IEEE Transactions on Microwave Theory and Techniques, Vol. 60, No. 6, 2012, pp. 1735-1742. http://dx.doi.org/10.1109/TMTT.2012.2189231

[7] W. Li, “A Narrow-Passband and Frequency-Funable Microwave Photonic Filter Based on Phase-Modulation to Intensity-Modulation Conversion Using a Phase-Shifted,” IEEE Transactions on Microwave Theory and Techniques, Vol. 60, No. 5, 2012, pp.1287-1296. http://dx.doi.org/10.1109/TMTT.2012.2187678

[8] R. Tao, X. Feng, Y. Cao, Z. Li and B. O. Guan, “Tunable Microwave Photonic Notch Filter and Bandpass Filter Based on High-Birefringence Fiber-Bragg-Grating-Based Fabry-Perot Cavity,” IEEE Photonics Technology Letters, Vol. 24, No. 20, 2012, pp. 1805-1808. http://dx.doi.org/10.1109/LPT.2012.2216258

[9] H. Terauchi, A. Tokunaga, N. Horaguchi and A. Maruta, "Design of All-Optical NRZ-OOK/RZ-QPSK Modulation 
Format Converter by Use of Cross Phase Modulation in Optical Fiber," OptoElectronics and Communications Conference, July 2012, pp. 899-900.

[10] Y. Du and G. P. Zhang, "Photonic Data Selector Based on Cross-Phase Modulation in a Highly Nonlinear Fiber," In Photo and Optoelectron, May 2012, pp. 1-3.

[11] X. Wu, X. Zheng, S. Li, H. Wen, Y. Guo and H. Zhang, "Multisideband Detection Radio-Over-Fiber Link with Phase Modulation and Fiber Demodulation for Vector Signal Transmission,” IEEE Photonics Technology Letters, Vol. 22, 2010 pp. 76-78.

http://dx.doi.org/10.1109/LPT.2009.2036145

[12] B. Yang, X. Jin, H. Chi, X. Zhang, S. Zheng, S. Zou, H. Chen, E. Tangdiongga and T. Koonen, "Optically Tuna- ble Frequency-Doubling Brillouin Optoelectronic Oscillator with Carrier Phase-Shifted Double Sideband Modulation,” IEEE Photonics Technology Letters, Vol. 24, 2012, pp. 1051-1053.

http://dx.doi.org/10.1109/LPT.2012.2194483

[13] R. Tao, X. Feng, Y. Cao, Z. Li and B. O. Guan, “ Widely Tunable Single Bandpass Microwave Photonic Filter Based on Phase Modulation and Simulated Brillouin Scattering," IEEE Photonics Technology Letters, Vol. 24, 2012, pp. 1097-1099.

http://dx.doi.org/10.1109/LPT.2012.2195486

[14] J. Yao, F. Zeng and Q. Wang, "Photonic Generation of Ultrawideband Signals,” IEEE/OSA Journal of Lightwave Technology, Vol. 25, 2007, pp. 3219-3235. 